\title{
Dimensional Changes of MOD Mold Cavity Corresponding to the Degree of Setting Expansion
}

\author{
Masahiro OHSAWA and Yoshihiko HAYASHI \\ Department of Endodontics and Operative Dentistry \\ Nagasaki University School of Dentistry \\ 1-7-1, Sakamoto, Nagasaki City, 852-8588, Japan
}

Received March 30, 1998/Accepted June 4, 1998

\begin{abstract}
The correlation between the degree of linear setting expansion and the dimensional changes in a mold space was examined. The dimensional changes in the mold were determined by investing the MOD-type wax pattern using a die-investing material of which the degree of the setting expansion was controlled by diluting a concentration of colloidal silica solution. The wax was eliminated in an electric furnace at $130^{\circ} \mathrm{C}$ and a fusible alloy, m.p. $48^{\circ} \mathrm{C}$, was cast into the mold space at room temperature. The dimensions of the wax pattern and the casting were compared. The degree of linear setting expansion was measured in a paper ring by means of a dial gauge. A high correlation was found between the dimensional changes of the mold space and the degree of the linear setting expansion. The expansion was highest at the axiogingival portion while those at both the axial dimension and the occlusal dimension were smallest. Linear regression lines $(\mathrm{Y}=\mathrm{AX}+\mathrm{B})$ revealed the differences in the expansion mode at both the external and internal portions to show a discrepancy in value $B$.
\end{abstract}

Key words: Effective setting expansion, MOD mold space, Die-investing material

\section{INTRODUCTION}

Modern dental investments have expansive properties, allowing for setting and thermal expansion to compensate for any casting shrinkage of the alloy used. Measurement of setting expansion is usually performed under a standardized test method, for example A.D.A.S. No2, 4.3.6., without the presence of a wax pattern inside the investment mass. The standardized method is thus suitable for a quality check of the materials. However, it has been reported that the degree of setting expansion in a mold (effective setting expansion, ESE) does not coincide with the value determined by the standardized method (linear setting expansion, LSE). Furthermore, the ESE at the internal (core) portion is larger than that at the external portion ${ }^{1)}$. It is still not clear how the magnitude of the setting expansion affects the dimensional changes of the mold space (wax pattern).

A gypsum-bonded cristobalite investment, commonly used for fabrication of inlays and crowns, exhibits at most a $1 \%$ setting expansion. The setting expansion of the die-investing material, however, mixed with a colloidal silica solution (CSS), depends on the concentration of CSS. A wide range of setting expansions ranging from $0.5 \%$ to $2.5 \%$ is obtained by diluting CSS and maintaining a constant $\mathrm{L} / \mathrm{P}$ ratio.

The aim of this study was thus to examine the correlation between the degree of 
setting expansion and the dimensional changes in the mold space (wax pattern), especially under a higher setting expansion which facilitated study of the correlation between the ESE and the LSE.

\section{MATERIALS AND METHODS}

The plate-type MOD wax patterns were prepared using a metal split mold and the press molding technique ${ }^{2)}$ with an indirect inlay wax (Violet Inlay Wax, GC, Tokyo, Japan). The wax patterns were left in the mold overnight in order to release any internal stress. The dimension of the wax pattern was measured with a traveling microscope equipped with a digital counter (Measurescope MM-11, Nikon, Tokyo, Japan). Heat generation by the light source of the microscope was considered to be small enough not to cause any dimensional changes of the wax pattern during measurement. The axio-pulpal line of the pattern was chosen as a fiducial line for measurement. The positioning of the pattern on the microscope stage was performed under $\times 10$ magnification and the dimension of each portion was determined under $\times 50$ magnification. The average dimensions of the wax pattern are illustrated in a rounded number at Fig. 1.

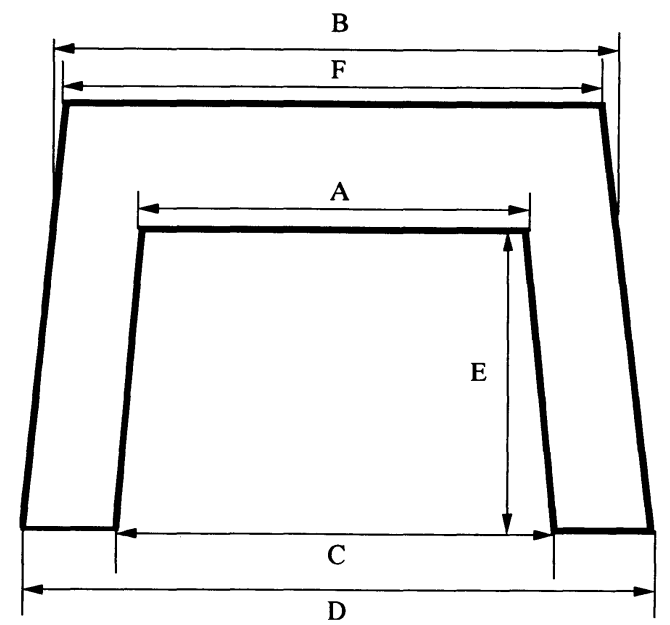

Fig. 1 Measured portion and dimensions of wax pattern

A: $9.32 \mathrm{~mm}$ Axiopulpal dimension

B: $13.54 \mathrm{~mm}$ Outer Axiopulpal dimension

C: $10.09 \mathrm{~mm}$ Axiogingival dimension

D: $14.19 \mathrm{~mm}$ Outer Axiogingival Dimension

E: $7.03 \mathrm{~mm}$ Axial dimension

$\mathrm{F}$ : $13.11 \mathrm{~mm}$ Occlusal dimension

$2 \mathrm{~mm}$ in thickness of wax pattern 
A roll of ceramic liner, measuring $0.7 \mathrm{~mm}$ thickness (Ceramic Ribbon, Noritake, Nagoya, Japan) was trimmed to $175 \mathrm{~mm}$ in length and $35 \mathrm{~mm}$ in width. It was held between two sheets and placed firmly inside the casting ring, $29 \mathrm{~mm}$ in inner diameter and $38 \mathrm{~mm}$ in height. A piece of wax (Ready Casting Wax, GC, Tokyo, Japan), $2 \mathrm{~mm}$ in diameter and $8 \mathrm{~mm}$ in length, was attached to the middle part of occlusal portion as a sprue while the other end of the sprue was fused to the top of the metal crucible former.

A die-investing material (Real Vest, Shofu, Kyoto, Japan) was employed as an investing medium and the accessory CSS was then diluted with water to generate a range of concentrations. The selected concentrations were 10,20 and $25 \%$. The $\mathrm{L} / \mathrm{P}$ ratio was 0.24 . Powder and water were first hand-mixed for $10 \mathrm{sec}$ and then mechanically mixed (Vacuum Mixer, Morita, Osaka, Japan) for $45 \mathrm{sec}$. The mixture was then poured into the casting ring and the patterns were inserted into the slurry. The position of the wax pattern in the casting ring is illustrated in Fig. 2.

Approximately 3 hours after investing, the molds were transferred into an electric furnace (accu-Therm 150, Jelenko, USA) in which the temperature was controlled at $130^{\circ} \mathrm{C}$ for approximately 2 hours in order to eliminate the wax from the mold ${ }^{3)}$. The molds were then bench-cooled to room temperature.

The fusible alloy (U-alloy, Asahi Metal, Osaka, Japan), with a melting point of $47^{\circ} \mathrm{C}$, was employed as a casting alloy. The alloy was melted in a hot water bath at approximately $75^{\circ} \mathrm{C}$. A centrifugal-type casting machine was used with a spring activation of two turns. Using stone pliers, the molds were broken into small pieces con-

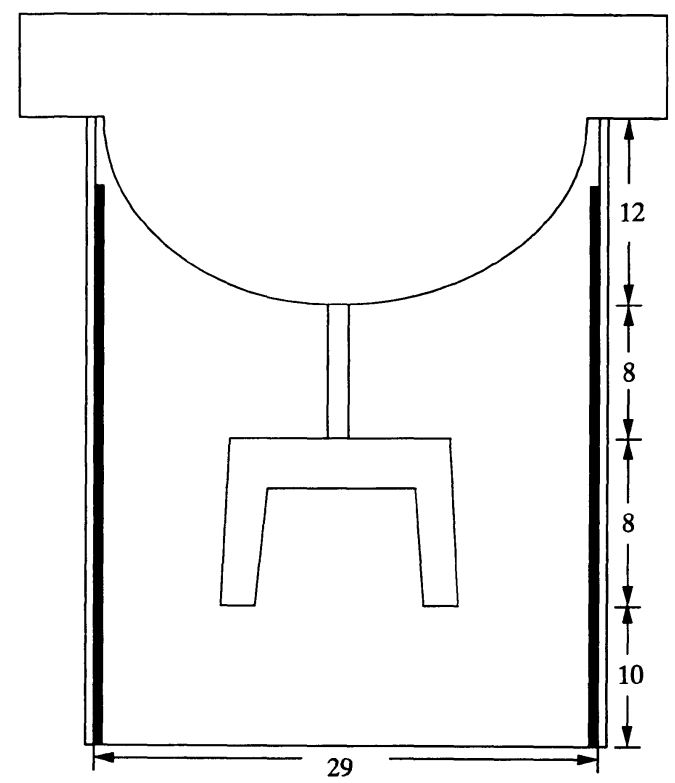

Fig. 2 Position of wax pattern in casting ring (unit: $\mathrm{mm}$ ) 
taining the castings and were then immersed into a dental stone dissolving solution (Super Melt, Shofu, Kyoto, Japan).

The dimensions of the castings were measured in the same manner as discribed for the wax pattern measurement. Dimensional differences between castings and wax patterns were calculated and expressed as a percentage.

The LSE of the die investment material was determined as follows ${ }^{4)}$ : slurries mixed at the above mentioned concentrations were poured into rings of waxed paper which had the same dimension as that of the casting ring. A plastic disc, measuring $20 \mathrm{~mm}$ in diameter and $2 \mathrm{~mm}$ in thickness, was placed on top of each slurry. The tip of the dial gauge (Mitutoyo, Tokyo, Japan) came into contact with the disc just after the gloss of the slurry had disappeared. All measurements continued for 2 hours.

\section{RESULTS}

The degree of LSE varied from $1.14 \%$ to $2.03 \%$ as listed in Table 1 . The degree of setting expansion showed an almost linear relation to the range of the selected concentration of CSS.

The dimensional changes in the resultant castings which represented the dimensions of the mold space are listed in Table 2. The specimen numbers were not necessarily the same for each portion due to small casting defects or damage caused when breaking the mold with stone pliers.

As a matter of course, the dimensions at each portion of the mold space for S10 through S25 increased in accordance with the degree of LSE. High correlations and linear regression lines were found between the ESE and the LSE as shown in Fig. 3.

\begin{tabular}{ccc} 
Table 1 & \multicolumn{2}{c}{ Linear setting expansion $(\mathrm{n}=3)$} \\
\hline Code & $\begin{array}{c}\text { Concentration } \\
\text { of CSS }(\%)\end{array}$ & Setting Expansion \\
\hline S10 & 10 & $1.14(0.04)$ \\
S20 & 20 & $1.67(0.02)$ \\
S25 & 25 & $2.03(0.04)$ \\
\hline \multicolumn{4}{c}{ Mean (S.D.) }
\end{tabular}

Table 2 Dimensional changes of mold space (\%)

\begin{tabular}{cccc}
\hline & S10 & S20 & S25 \\
\hline A & $1.03 \pm 0.12(9)$ & $1.37 \pm 0.10(9)$ & $1.60 \pm 0.10(9)$ \\
B & $0.81 \pm 0.08(9)$ & $1.12 \pm 0.09(12)$ & $1.28 \pm 0.08(9)$ \\
C & $1.31 \pm 0.07(9)$ & $1.78 \pm 0.12(10)$ & $2.00 \pm 0.09(8)$ \\
D & $1.06 \pm 0.08(9)$ & $1.37 \pm 0.07(9)$ & $1.58 \pm 0.08(8)$ \\
E & $0.77 \pm 0.05(9)$ & $1.14 \pm 0.05(9)$ & $1.31 \pm 0.10(9)$ \\
F & $0.68 \pm 0.04(8)$ & $1.03 \pm 0.07(11)$ & $1.16 \pm 0.09(9)$ \\
\hline & & &
\end{tabular}

( ): numbers of the tested specimen 


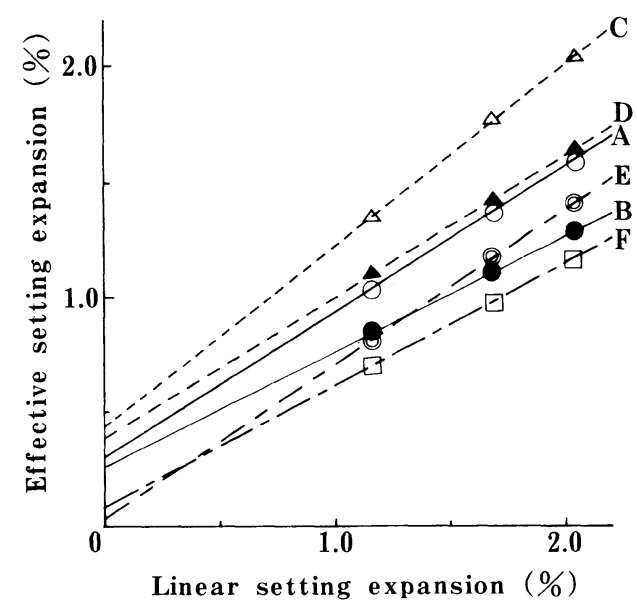

Fig. 3 Linear regression lines and coefficient of correlations
A: $\mathrm{ESE}=0.43 \mathrm{LSE}+0.30 \quad \mathrm{r}=0.910$
$\mathrm{B}: \mathrm{ESE}=0.51 \mathrm{LSE}+0.25 \mathrm{r}=0.912$
$\mathrm{C}: \mathrm{ESE}=0.79 \mathrm{LSE}+0.43 \quad \mathrm{r}=0.942$
$\mathrm{D}: \mathrm{ESE}=0.62 \mathrm{LSE}+0.38 \quad \mathrm{r}=0.888$
$\mathrm{E}: \mathrm{ESE}=0.68 \mathrm{LSE}+0.03 \quad \mathrm{r}=0.982$
$\mathrm{F}: \mathrm{ESE}=0.54 \mathrm{LSE}+0.08 \quad \mathrm{r}=0.919$

Table 3 Ratio of effective setting expansion and linear setting expansion

\begin{tabular}{llll}
\hline & S10 & S20 & S25 \\
\hline A & 0.90 & 0.82 & 0.79 \\
B & 0.71 & 0.67 & 0.63 \\
C & 1.15 & 1.07 & 0.99 \\
D & 0.93 & 0.82 & 0.78 \\
E & 0.68 & 0.68 & 0.65 \\
F & 0.60 & 0.62 & 0.57 \\
\hline
\end{tabular}

On the linear regression line $(\mathrm{Y}=\mathrm{AX}+\mathrm{B})$, each value of $\mathrm{B}$ on an individual line was devided into two groups: the first groups were axiogingival and outer axiogingival, and axiopulpal and outer axiopulpal, in which the values of $\mathrm{B}$ were larger than 0.2 ; and the second groups were axial dimension and occlusal width, in which values of $B$ were close to zero.

The values of the ESE in each portion were then divided by those of the LSE. Judging from this ratio, the degree of LSE was generally larger than that of ESE, except for 2 portions as shown in Table 3 . The index ratio at the axiogingival dimension was the largest while that at the occlusal dimension was the smallest throughout the 3 groups. 


\section{DISCUSSION}

In this study, a die-investing material was used as the investing medium because the serial setting expansion was obtained by diluting the CSS, rather than by altering the $\mathrm{L} / \mathrm{P}$ ratio. The LSE of $2.5 \%$ was obtained when mixed with undiluted (100\%) CSS, and the degree of setting expansion was subsequently linear until $30 \%$ concentration $^{5)}$. The higher the concentration of CSS, the harder and stronger the set material, and as a result, the $\mathrm{S} 30$ group was not tested because the molds could not be broken into small pieces with the stone pliers. Water absorption by the liner was confirmed to be negligible in another study ${ }^{6}$.

According to the manufacturer's information on the fusible alloy ${ }^{7)}$, the composition of this alloy was $\mathrm{Cd}, \mathrm{Pb}, \mathrm{Sn}$ and $\mathrm{Bi}$ while the dimensional change after casting was negligible (less than $0.001 \%$ ). This alloy was so frail that the casting could only be taken out from the mold by shattering the mold materials with stone pliers, thus damaging or deforming the casting. The small block of investment containing the casting was thus immersed in a stone melting solution.

The ESE was larger than the LSE at 2 portions among 3 groups, especially at the axiogingival dimension. This portion originally showed a huge expansion, in comparison to the other portions. Davis and Kennedy ${ }^{3)}$ reported a $0.6 \%$ expansion for this portion even though the setting reaction was inhibited by addition of algin to the gypsum-bonded investment. The regression line (Fig. 3, C) of the present study also indicated an approximate $0.4 \%$ expansion at the axiogingival dimension when the setting expansion was assumed to be zero.

Two modes of expansion are considered to be exerted, as Mahler and $\mathrm{Ady}^{1)}$ have already reported: 1) an external mode such as the occlusal dimension where the investment around the wax pattern expands outward away from the pattern, creating a space between the wax pattern and the investment, and 2) an internal mode, such as the axiopulpal-axiogingival area where the core portion of the investment expands, thus extending the wax pattern. Based on the regression lines $(\mathrm{Y}=\mathrm{AX}+\mathrm{B})$ of this study, the values of $B$ were different in the internal and external portions. As a result, the value for such external portions as the axial dimension and occlusal dimension were close to zero. The outer axiopulpal and outer axiogingival portions belonged to the external portions but they were affected by the core portion, thus their values were not close to zero.

\section{REFERENCES}

1) Mahler, D. B. and Ady, A. B.: The influence of various factors on the effective setting expansion of casting investments, J Prosthet Dent 13(2):365-373, 1963.

2) Fusayama, T.: Factors and technique of precision casting, Part 1, J Prosthet Dent 9(3): 468-485, 1959.

3) Davis, D. R. and Kennedy, M. P.: Xeroradiographic determination of effective setting expansion of a cristobalite investment, Dent Mater 6(1):29-34, 1990.

4) Jorgensen, K. D.: Study of the setting expansion of gypsum, Acta Odontl Scand 21 : 227$257,1963$. 
5) Ohsawa, M., Shibuya, M. and Matsumoto, H.: Study on casting dimensions, Part 2 Differences between inside and outside dimension of the resultant dies, Japan $J$ Conserv Dent 33(1): 112-115, 1990. (in Japanese)

6) Ikeda, T., Shibuya, M., Ohsawa, M. and Matsumoto, H.: Study on effects of various casting liners on dimensional changes of mold, Japan J Conserv Dent 35(5) : 1265-1274, 1993. (in Japanese)

7) U-alloy series, Osaka Asahi Metal MFG. CO., LTD. p.3, 6. 